\title{
Fatores estressores em unidade de terapia intensiva neonatal: percepções familiares
}

\author{
Stress factors in the neonatal intensive care unit: \\ family perceptions
}

\author{
Roberta Tognollo Borotta Uema' $\bullet$ Bruna Caroline Rodrigues $^{2} \bullet$ Gabrieli Patricio Rissi $^{3}$ \\ Larissa Carolina Segantini Felipin ${ }^{4} \bullet$ Bianca Machado Cruz Shibukawa ${ }^{5} \bullet$ Muriel Fernanda de Lima $^{6}$ \\ leda Harumi Higarashi ${ }^{7}$
}

\begin{abstract}
RESUMO
Objetivo: identificar os fatores estressores vivenciados pelos pais de recém-nascidos hospitalizados em unidade de terapia intensiva neonatal pela aplicação de um instrumento baseado na escala Parental Stress Scale: Neonatal Intensive Care Unit. Método: pesquisa qualitativa realizada com a aplicação de um instrumento baseado na escala Parental Stress Scale: Neonatal Intensive Care Unit, com sete mães e dois pais de bebês internados em um hospital universitário, entre os meses de fevereiro e maio de 2018.A análise dos dados foi realizada por estatística descritiva. Resultados: os dados encontrados demonstraram que existiam diversos fatores de estresse dentro da unidade, incluindo o próprio ambiente da unidade e que, o período de hospitalização impactava na percepção que os pais dos bebês tinham sobre seu papel. Conclusão: os fatores de estresse identificados foram relacionados ao ambiente da unidade de terapia intensiva neonatal e as situações que ocorreram em virtude da hospitalização precoce do bebê. Cabe à equipe multidisciplinar, auxiliar desde o primeiro momento para que as famílias vivenciem esse processo de uma forma menos traumatizante e mais humanizada.

Palavras-chave: Estresse psicológico; Relações profissional-família; Unidades de terapia intensiva neonatal; Família; Recém-nascido.
\end{abstract}

\begin{abstract}
Objective: to identify the stressors experienced by parents of newborns hospitalized in a neonatal intensive care unit by applying an instrument based on the Parental Stress Scale: Neonatal Intensive Care Unit. Method: qualitative research carried out with the application of an instrument based on the Parental Stress Scale: Neonatal Intensive Care Unit, with seven mothers and two parents of babies admitted to a university hospital, between the months of February and May 2018. The analysis of data was performed using descriptive statistics. Results: the data found demonstrated that there were several stress factors within the unit, including the unit's own environment and that the hospitalization period impacted the perception that the babies' parents had about their role. Conclusion: the stress factors identified were related to the environment of the neonatal intensive care unit and the situations that occurred due to the baby's early hospitalization. It is up to the multidisciplinary team to assist from the first moment so that families experience this process in a less traumatic and more humanized way.

Keywords: Stress, Psychological; Professional-Family Relations; Intensive Care Units, Neonatal; Family; Infant, Newborn.
\end{abstract}

NOTA

I Enfermeira do Hospital Santa Casa de Maringá. Doutora em Enfermagem pelo Programa de Pós-Graduação em Enfermagem da Universidade Estadual de Maringá. Maringá, Paraná, Brasil.

2 Enfermeira do Hospital Municipal de Maringá. Doutora em Enfermagem pelo Programa de Pós-Graduação em Enfermagem da Universidade Estadual de Maringá. Maringá, Paraná, Brasil.

3 Enfermeira. Docente de Enfermagem na Universidade Unicesumar. Doutoranda em Enfermagem pelo Programa de Pós-Graduação em Enfermagem da Universidade Estadual de Maringá. Maringá, Paraná, Brasil.

4 Enfermeira. Doutoranda em Enfermagem pelo Programa de Pós-Graduação em Enfermagem da Universidade Estadual de Maringá. Maringá, Paraná, Brasil.

5 Enfermeira. Doutoranda em Enfermagem pelo Programa de Pós-Graduação em Enfermagem da Universidade Estadual de Maringá. Maringá, Paraná, Brasil.

6 Enfermeira. Docente de Enfermagem na Universidade Estadual do Paraná. Doutora em Enfermagem pelo Programa de Pós-Graduação em Enfermagem da Universidade Estadual de Maringá. Maringá, Paraná, Brasil.

7 Enfermeira. Docente de Enfermagem na Universidade Estadual de Maringá. Doutora em Educação pela Universidade Federal de São Carlos. Maringá, Paraná, Brasil.

Autor correspondente: Gabrieli Patricio Rissi.Avenida Colombo, 5790. Jardim Universitário. Maringá, Paraná, Brasil. CEP 87020-900. Contato: (44)99856-7785. E-mail: gabrielirissi@gmail.com 


\section{INTRODUÇÃO}

Estima-se que nasçam cerca de 15 milhões de bebês prematuros no mundo e atualmente pode-se perceber uma mudança importante no cenário da assistência em unidade de terapia intensiva neonatal (UTIN), pois com os avanços da tecnologia salvam-se bebês cada vez mais prematuros, até então considerados inviáveis ${ }^{(1-2)}$.

A hospitalização de um filho em UTIN, gera uma experiência estressante para os pais, que quando somada aos aspectos particulares da prematuridade, tende a aumentar consideravelmente, uma vez que estes convivem com a possibilidade do bebê não sobreviver e do risco aumentado de complicações a longo prazo $^{(3)}$.

O próprio ambiente da UTIN, rodeado de sons e luzes desconhecidas, o fato de ver seu bebê conectado a tubos e cateteres, o próprio aspecto frágil que o prematuro apresenta, a exposição aos procedimentos e intervenções, somado a frustração da idealização do nascimento de um bebê grande e saudável, pode aumentar o estresse vivenciado pelos pais ${ }^{(3-4)}$.

O estresse vivenciado nesse período pode levar a quadros depressivos, transtornos de ansiedade, fadiga e distúrbios de sono, que podem perdurar até à alta hospitalar do bebê e depois dela, comprometendo a criação de vínculo entre pais e filhos, e o desenvolvimento neurológico desse recém-nascido $(\mathrm{RN})^{(5)}$. $\mathrm{O}$ estresse dos pais que apresentam sintomas depressivos, influencia diretamente no desenvolvimento social, comportamental e funcional do bebê $\hat{e}^{(6)}$.

Escalas que tem por objetivo mensurar o nível de estresse vivenciado pelos pais, a fim de traçar estratégias que auxiliem a superar este momento, são uma das alternativas que ajudam na identificação e no enfrentamento dessas situações. Uma delas é a Parental Stress Scale: Neonatal Intensive Care Unit (PSS: NICU), criada em 1993 na língua inglesa e posteriormente traduzida e validada em 2012 para a língua portuguesa do $\mathrm{Brasil}^{(7)}$.

Identificar os fatores estressores que permeiam a internação do RN internado em UTIN e são vivenciados pelos pais, é uma forma de auxiliar a melhorar o estabelecimento do vínculo, saúde física e mental, além de estimular o desenvolvimento posterior desse bebê $\hat{e}^{(2)}$. Em virtude disso, objetivou-se identificar os fatores estressores vivenciados pelos pais de recém-nascidos hospitalizados em unidade de terapia intensiva neonatal, com a finalidade de responder o seguinte questionamento: Quais são os fatores estressores vivenciados pelos pais de recém-nascidos hospitalizados na unidade de terapia intensiva neonatal?

\section{MÉTODO}

Trata-se de uma pesquisa qualitativa de abordagem descritiva. $O$ estudo foi realizado com nove pais de bebês internados em uma UTIN, sendo estes sete mães e dois pais. A unidade faz parte de um Hospital Universitário situado no sul Brasil, que conta com 10 leitos neonatais, sendo quatro de cuidados intermediários e seis leitos de cuidados intensivos.

Utilizou-se como critérios de inclusão pais ou mães de bebês nascidos prematuros, com idade gestacional abaixo de 36 semanas e 6 dias, e ter idade igual ou superior a 18 anos. Foram excluídos do estudo, familiares que possuíam histórico de doença psiquiátrica ou física, que relatassem uso de medicamentos para ansiedade ou depressão, pois estes já possuem comorbidades que poderiam afetar os resultados deste estudo.

Também foram excluídos familiares analfabetos, visto que, o instrumento utilizado era autopreenchido. Os familiares de bebês que fossem a óbito durante o período de coleta também foram excluídos.

A coleta de dados ocorreu por meio da aplicação de um instrumento baseado na escala Parental Stress Scale: Neonatal Intensive Care Unit ${ }^{(7)}$. A escolha da escala referida, justifica-se pelo fato de a mesma avaliar parâmetros ambientais, de relacionamento e especificamente à aparência do bebê prematuro, que muitas vezes se torna um fator desencadeador de ansiedade na família Os dados foram coletados entre os meses de fevereiro e maio de 2018.

Para $\circ$ recrutamento dos participantes, abordavamse os familiares durante seu período de permanência na unidade neonatal, nos horários liberados para visita estendida.Após explanação inicial dos objetivos do estudo, se o mesmo cumprisse os critérios de inclusão, era encaminhado para uma antessala dentro da própria UTIN e instruído sobre o preenchimento do instrumento.

$O$ instrumento criado contava com uma primeira parte relacionada à busca de dados sócio demográficos composto por questões abertas e a segunda parte, composta por dez itens, os quais se dirigiam especificamente aos fatores estressores na visão dos pais. Cada item do instrumento contava com duas alternativas: "sim" e "não". As questões versavam sobre a aparência do bebê, a separação do filho(a) durante a internação, e o ambiente da UTIN.

Todos os preceitos éticos da Resolução 466/20I 2 do Conselho Nacional de Saúde foram contemplados, bem como a assinatura em Termo de Consentimento Livre e Esclarecido $(T C L E)^{(8)}$. A pesquisa foi aprovada pelo Comitê Permanente de Ética em Pesquisa Envolvendo Seres Humanos, sob parecer $n^{\circ} 2.092 .136$ e CAAE $n^{\circ}$ 66242617.4 .0000 .0104 .

\section{RESULTADOS}

Dos nove sujeitos abordados, sete eram mães e dois eram pais. A idade variou entre 30 e 45 anos, com média 
de 37 anos. Somente dois pais possuíam curso superior completo, quatro enquadravam-se no ensino fundamental três no médio completo. Cinco residiam no mesmo munício da unidade estudada e o restante morava nas cidades vizinhas com menos de $30 \mathrm{~km}$ de distância.

A maioria dos participantes referiram dificuldades de locomoção para chegar até a UTIN a fim de visitar seu bebê. Cinco, demoravam cerca de 30 minutos para chegar ao hospital, e o restante até uma hora. Sete possuíam outras crianças para cuidar em casa, porém relataram ter rede de apoio familiar para os cuidados com as crianças, e, portanto, conseguiam visitar seu bebê na unidade.

Dentro os itens que compunham o instrumento, o excesso de equipamentos dentro da UTIN (89\%), juntamente com a respiração anormal do bebê (67\%), agitação do mesmo (67\%), o bebê apresentar face de dor (67\%), e a cor do bebê (55\%) foram os fatores mais estressantes considerados pelos pais.

O fato de não estarem habituados a esse ambiente, e enxergar o bebê com uma aparência desconfortável, são situações que os deixaram com sentimentos desagradáveis. Ao mesmo tempo, a aparência frágil do bebê prematuro (11\%), com a pele friável, o baixo peso e o tamanho pequeno (22\%), não foi considerada como altamente estressantes.

Em relação aos cuidados comumente realizados ao nascimento do bebê, mas que dentro da unidade neonatal acaba sendo dificultado, percebeu-se que os pais identificaram como situações estressantes, aqueles momentos em que não conseguiam exercer seu papel de cuidador do bebê em cuidados básicos, como alimentar (55\%), dar colo (78\%) e sentir que de alguma forma estavam protegendo se filho (67\%).

A própria separação decorrente da hospitalização (67\%) e não conseguir amamentar nos primeiros dias de vida (55\%) também foram identificados como estressores. Em muitos casos, o bebê não consegue ser alimentado nos primeiros dias de vida ou então encontra-se em estado muito grave, impossibilitando o colo ou a posição canguru e isso também interfere nos sentimentos expressados pelos pais nesse período de internação.

Sobre a ambiência da UTIN, os resultados nos mostram que os monitores conectados ao bebê podem ser considerados os piores fatores de estresse (55\%), mais do que o fato destes estarem acoplados à ventilador mecânico (VM) (45\%).

O barulho constante (33\%), ver outros bebês internados $(33 \%)$ e a presença de outras pessoas dentro da UTIN (I I\%) foram os outros itens identificados como fatores de estresse.Ademais, salienta-se que $67 \%$ dos entrevistados relataram que o fato de não poderem proteger seu bebê da dor era algo altamente estressante.

Vivenciar a internação do seu filho junto com a hospitalização dos outros bebês não foi considerado como fator altamente estressor, visto que compartilham suas ansiedades com os outros familiares de bebês também internados, criando laços de amizade e redes de apoio compartilhadas pelos pais que estão imersos nessa realidade.

\section{DISCUSSÃO}

A unidade de terapia intensiva neonatal se configura como um ambiente altamente tecnológico, em que os avanços e a intervenção profissional, se voltam para a sobrevivência e recuperação do bebê, consequentemente, as condições de saúde ou doença são sobrepostas às demais necessidades, como apego e afeto ${ }^{(9)}$.

A UTIN coloca o bebê em diversas situações, procedimentos invasivos, luminosidade excessiva, ruídos, manipulação, interrupção constante de sono, ausência materna e a solidão da incubadora, cenário o qual gera uma conjuntura estressora não apenas aos bebês, mas também aos familiares que acompanham como expectadores ${ }^{(3-4,9)}$.

A transição da vida uterina para uma incubadora, apesar de ser crucial para a sobrevivência do bebê prematuro, influencia negativamente o desenvolvimento do recém-nascido, pois o ambiente da UTIN é geralmente inóspito e causa estímulos negativos e dolorosos ${ }^{(10)}$.

Para a família que acompanha esse processo, isso causa um alto nível de estresse, num primeiro momento por não compreender a linguagem complexa utilizada dentro da UTIN, e posteriormente por lidar com o sofrimento em estar longe do filho, o medo da perda e o sentimento de fracasso(II).

O fato dos pais presenciarem seu bebê passar por procedimentos dolorosos, ou encontrá-lo com face de dor e agitação, duas características que eminentemente indicam sinal de desconforto, é uma situação altamente estressante ${ }^{(12)}$. Apesar da maioria das instituições fazer uso de protocolos e recomendações para alívio da dor e do desconforto, ainda encontramos barreiras para que todos métodos sejam aplicados, causando não somente um alívio para o bebê, mas também para aqueles que estão próximos e vivenciando o internamento junto com ele ${ }^{(13)}$.

A separação do bebê durante o período de hospitalização, somado à limitação em poder alimentar, cuidar, segurar e o fato de não poder proteger seu bebê de intervenções que são necessárias à sua sobrevivência, mas que ao mesmo tempo, tornam-se dolorosas e desconfortáveis, é caracterizado pelos pais como situações altamente estressoras ${ }^{(4,13-14)}$.

Nota-se que esses sentimentos estão mais relacionados à figura materna, entretanto, encontrou-se que os pais também apresentam esse tipo de sentimento, fato 
que deve ser considerado pela equipe multidisciplinar da UTIN como estratégia de intervenção com os familiares $^{(15)}$. Quando relacionado à figura da mãe, estas relatam sentimento de perda de função, no sentido de possuir dificuldades em reconhecerem seus próprios filhos, impactando diretamente em seu desenvolvimento futuro ${ }^{(5,12)}$.

Sabe-se que quando a família é inclusa no cuidado da criança hospitalizada em longa permanência, a relação construída com a equipe multidisciplinar e como com seu próprio filho, é sabidamente melhor ${ }^{(2-4)}$. A integração da família no cuidado, propicia sensação de utilidade naquele período em que não podem levar seus filhos para casa, ao mesmo tempo que favorece criação de vínculo com a equipe, e fornece sensação de segurança para a futura alta hospitalar do bebê $\hat{e}^{(16)}$.

Corroborando com o descrito acima, entende-se que, quando os profissionais de saúde incluem as mães nos cuidados dos seus filhos, estas adquirem maior segurança e autonomia, sentindo-se mais competentes para cuidar do filho ${ }^{(12)}$. Os profissionais podem ajudar nesse processo de construção da autonomia materna, primeiramente exercendo $o$ acolhimento, a escuta ativa, orientando os cuidados compassivamente e num posterior momento, incentivá-la a fazer os cuidados sozinha ficando o profissional apenas na supervisão e orientação quando necessário(6).

O sentimento de impotência e inviabilidade de proteção contra a dor de seus filhos, consiste em algo altamente estressante para os pais. Um estudo multicêntrico realizado na América do Sul, também reporta que, concomitante a sensação de falta de proteção, não poder alimentar o bebê e não saber como ajudá-lo durante o período de hospitalização em UTIN, constituem-se como situações potencialmente estressoras e prejudiciais à sua saúde mental ${ }^{(17)}$.

Diversas estratégias devem ser consideradas no apoio aos pais dos prematuros, frente à ansiedade em lidar com a condição do seu bebê hospitalizado, tais como facilitar 0 acesso às redes de apoio, inclusão nos cuidados, fornecer informações adequadas e incentivar o contato pele a pele. ${ }^{(18)} \mathrm{Na}$ neonatologia, os grupos de apoio como estratégias de terapia tem auxiliado os pais a lidar com a ansiedade decorrente da internação do bebê na UTIN ${ }^{(19)}$.

A relação da equipe multidisciplinar com a família, deve ser pautada no respeito uma vez que a internação do recém-nascido na UTIN gera uma situação de crise dentro do ambiente familiar ${ }^{(12,15)}$. Dessa forma, apesar das dificuldades que aparecem nesse processo, a equipe multidisciplinar, torna-se o elo entre os pais e os bebês. Essa promoção de vínculo auxilia no desenvolvimento emocional entre família e neonato, e é considerado pelos pais, como o sentimento de maior gratificação( ${ }^{(20)}$.

\section{CONCLUSÃO}

Os fatores estressores dos pais de bebês internados em unidades de terapia intensiva neonatal mais significativos neste estudo foram o excesso de equipamentos dentro da UTIN, respiração anormal, agitação, face de dor e a coloração do bebê. Os sentimentos de impotência frente às necessidades dos filhos são geradores de frustração nos pais, portanto, cabe a equipe multidisciplinar estimular a interação dos pais com o bebê, favorecendo a criação e fortalecimento de vínculo entre os familiares e seu concepto.

A relevância deste estudo se traduz na humanização do cuidado ao recém-nascido prematuro e seus familiares, uma vez que, a identificação dos fatores estressantes aos pais favorece a criação de estratégias pela equipe multidisciplinar que auxiliem no acolhimento humanizado dos familiares, amenizando o sofrimento pela hospitalização prematura de seus filhos. 


\section{REFERÊNCIAS}

I. World Health Organization (WHO). Preterm birth. [Internet]. 2018 [cited 2020 July 9]. Available from: https://www. who.int/en/news-room/fact-sheets/detail/preterm-birth

2. Kegler JJ, Neves ET, Silva AM, Jantsch LB, Bertoldo CS, Silva $\mathrm{JH}$. Stress in parents of newborns in a Neonatal Intensive Care Unit. Esc. Anna Nery Rev. Enferm. [Internet]. 2019 [cited 2019 Nov 12]; 23(I):e20 I80 I 78.Available from: https:// www.scielo.br/scielo.php?script=sci_arttext\&pid =SI4I4814520190001002II

3. Feng YY, Korale-Liyanage S, Jarde A, McDonald SD. Psychological or educational eHealth interventions on depression, anxiety or stress following preterm birth: a systematic review. J. Reprod. Infant Psychol. [Internet]. 2020 [cited 2020 July 9]; I-I3. Available from: https://pubmed.ncbi. nlm.nih. gov/32336122/

4. Bonacquisti A, Geller PA, Patterson CA. Maternal depression, anxiety, stress, and maternal-infant attachment in the neonatal intensive care unit. J. Reprod. Infant Psychol. [Internet]. 2020 [cited 2020 July 9]; 38(3):297-3 I0.Available from: https://pubmed.ncbi.nlm.nih.gov/31795733/

5. Santos LF, Souza IA, Mutti CF, Santos NSS, Oliveira LMAC. Forces interfering in the mothering process in a neonatal intensive therapy unit. Texto \& contexto enferm. [Internet]. 2017 [cited 2019 Oct 25]; 26(3):el2600I6. Available from: https://www.scielo.br/scielo.php?script=sci_arttext\&pid= S0104-0707201700030032I

6. Loewenstein K, Barroso J, Phillips S. The Experiences of Parents in the Neonatal Intensive Care Unit An Integrative Review of Qualitative Studies Within the Transactional Model of Stress and Coping. J. perinat. neonatal nurs. [Internet]. 2019 [cited 2020 July 9]; 33(4):340-349.Available from: https://pubmed.ncbi.nlm.nih.gov/ 31651628/

7. Souza SR, Dupas G, Balieiro MMFG. Cultural adaptation and validation for the Portuguese language of the Parental Stress Scale: Neonatal Intensive Care Unit (PSS:NICU). Acta paul. enferm. [Internet]. 2012 [cited 2019 Oct 25]; 25(2): I7I-6. Available from: https://www.scielo.br/ scielo.php?script=sci_arttext\&pid=SO I 03-2 I 0020 I 2000200003\&lang=en

8. Ministério da Saúde (BR). Conselho Nacional de Saúde. Di -retrizes e normas regulamentadoras de pesquisa envolvendo seres humanos. Resolução n. 466, de 12 de dezembro de 2012. Brasília; 2012.

9. Pinheiro MR, Carr AMG. The effectiveness of the kangaroo mother method in comparison of conventional care in a Neonatal UTI. Braz. J. Health Rev. [Internet]. 2019 [cited 2019 Oct 25]; 2(2):1039-1048. Available from: http://brazilianjournals.com/index.php/BJHR/article/ view/I295/ I I70.

10. Baseggio DB, Dias MPS, Brusque SR, Donelli TMS, Mendes P. Mothers and premature infants experiences during neonatal hospitalization. Temas psicol. (Online). [Internet]. 2017 [cited 2020 July 9]; 25(I):I53-167. Available from: http:// pepsic.bvsalud.org/scielo.php?script=sci_arttext\&pid= SI4I3-389X20170001000I0\&Ing=pt.

I I. Brigida ECSS, Andrade GCF, Araújo RBS. Humanization of nursing in the Neonatal Intensive Theapy Unit (NICU). J. Specialist. [Internet]. 2018 [cited 2019 Oct 25]; 2(2): I-I2. Available from: https://docplayer.com.br//3472568I-Humanization-of-nursing-in-the-neonatal-intensive-theapy-unit-nicu-humanizacao-da-enfermagem-na-unidade-de-terapia-intensiva-neonatal-utin.html

12. Soares MFE, Chaves AVG, Morais APS, Rabelo MZS, Rodrigues LN, Chaves EMC. Newborn's pain under the mother's perception. Rev. dor [Internet]. 2017 [cited 2020 July 09]; I8(4):338-34I. Available from: http://www.scielo.br/ scielo.php?script=sci_arttext\&pid=SI 806-00 I 3201700040 0338\&lng=en.

13. Maciel HIA, Costa MF, Costa ACL, Marcatto JO, Manzo BF, Bueno M. Pharmacological and nonpharmacological measures of pain management and treatment among neonates. Rev. bras. ter. intensiva. [Internet]. 2019 [cited 2020 July 9]; 3I (I):2I-26. Available from: https:// .scielo.br/scielo.php?script=sci_arttext\&pid=S0103-507X201900010002 I

14. Baía I, Amorim M, Silva S, Kelly-Irving M, Freitas C, Alves E. Parenting very preterm infants and stress in Neonatal Intensive Care Units. Early Hum Dev. [Internet]. 2016 [cited 2019 Oct 29]; 101:3-9. Available from: https://www.ncbi. nlm. nih.gov/pubmed/27393922 I5.

15. Carvalho LS, Pereira CMC. Psychological reactions of parents for hospitalization of premature infants in the neonatal ICU. Rev. SBPH [Internet]. 2017 [cited 2020 July 9]; 20(2):I0I-I22. Available from: http:// pepsic.bvsalud.org/scielo.php?script=sci_arttext\&pi$d=S|5|$ 6-085820 I 7000200007 \&lng=pt.

16. Azevêdo AVS, Lançoni Júnior AC, Crepaldi MA. Nursing team, family and hospitalized child interaction: an integrative review. Ciênc. Saúde Colet. [Internet]. 2017 [cited 2020 July 9]; 22(II):3653-3666. Available from: https://www.scielo.br/scielo.php?script=sci_arttext\&pi$d=S|4| 3-8|2320| 702|| 03653$

17. Chourasia N, Surianarayanan P, Adhisivam B,Vishnu Bhat B. NICU admissions and maternal stress levels. Indian j. pediatr. [Internet]. 2013 [cited 2019 Dec II]; 80(5):3804. Available from: https://www.ncbi. nlm.nih.gov/pubmed/231804I3

18. Balbino FS, Yamanaka Cl, Balieiro MMFG, Mandetta MA. Parent's support group as a transforming experience for families at a neonatal unit. Esc Anna Nery Ver. Enferm. [Internet]. 2015 [cited 2019 Dec II]; 19(2): 297302. Available from: https://www.scielo.br/scielo.php?pi$\mathrm{d}=\mathrm{S}|4| 4-8|4520| 5000200297 \&$ script=sci_abstract \&t|$\mathrm{ng}=\mathrm{pt}$

19. Correia LA, Rocha LLB, Dittz ES. Contributions of the occupational therapy group to the anxiety level of mothers with premature newborns admitted in the neonatal intensive care 
units. Cad. Bras. Ter. Ocup. [Internet]. 2019 [cited 2019 Dec 14]; 27(3):574-583. Available from: https://www.scielo.br/scielo.php?pid=\$2526-89102019000300574\&script=sci_arttext

20. Sousa SC, Medino MS, Benevides KGCB, Ibiapina AS, Ataíde $K M N$. Strengthening the link between the family and the premature newborn. Rev. enferm. UFPE on line. [Internet].
2019 [cited 2019 Dec 14]; 13(2):298-306. Available from: https://periodicos.ufpe.br/revistas/revistaenfermagem/ article/view/236820/3I 268.

Recebido: 2020-07-20

Aceito: 2020-09-02 